\title{
Selenium and host defence towards viruses
}

\author{
Melinda A. Beck \\ Pediatrics and Nutrition, 535 Burnett-Womack Building, CB\# 7220, University of North Carolina at Chapel Hill, Chapel Hill, \\ NC 27599-7220, USA
}

\begin{abstract}
The association between viral disease and nutrition has long been thought to be due to effects on the host immune system. This theory suggests that when a host is malnourished, the immune system is compromised, and thus increased susceptibility to viral infection will occur. However, the virus itself may also be affected by the nutritional status of the host. We have demonstrated that a normally-benign strain of coxsackievirus B3 (CVB3/0) becomes virulent in either Sedeficient or vitamin E-deficient mice. Although the deficient animals are immunosuppressed, the virus itself is also altered. Six nucleotide changes were found in the virus that replicated in the deficient mice, and once these mutations occurred, even mice with normal nutrition became susceptible to disease. Thus, the nutritional status of the host was able to transform an avirulent virus into a virulent one due to genomic changes in the virus. We believe that a common mechanism of oxidative stress is the underlying cause of the genetic changes. Both vitamin $\mathrm{E}$ and Se act as antioxidants, and benign virus inoculated into GSH peroxidase (EC 1.11.1.9)-knockout mice will also convert to virulence due to genomic changes. Our work points to the importance of host nutrition during a viral disease, not only from the perspective of the host, but from the perspective of the viral pathogen as well.
\end{abstract}

Coxsackievirus: Nutritional deficiency: Oxidative stress: Selenium: Viral mutation

It has long been known that nutritional deficiency of the host leads to increased susceptibility to infectious disease (Scrimshaw et al. 1968; Scrimshaw, 1975). Throughout history periods of famine were often accompanied by epidemics of infectious diseases. Nutritionally-deficient hosts frequently develop more severe pathology following a viral infection when compared with well-nourished individuals. For example, rotavirus infection of malnourished children frequently leads to severe diarrhoea and dehydration with a high rate of mortality, whereas rotavirus infection of wellnourished children leads to mild diarrhoea.

The relationship between malnutrition and increased susceptibility to infectious disease has been thought of as an interaction between the host's immune system and the pathogen; i.e. the malnutrition causes the immune system to dysfunction, leading to increased susceptibility to infectious pathogens. This process can be illustrated as follows:

$$
\begin{gathered}
\text { host malnutrition } \\
\downarrow \\
\text { decreased immunity } \\
\downarrow
\end{gathered}
$$

increased susceptibility to viral pathogenesis
However, our recent work has suggested that an alternative to this model is possible. We have found that the nutritional status of the host not only affects the host, but can have a direct effect on the viral pathogen as well. In particular, a normally-benign strain of coxsackievirus B3 (CVB3/0) will become virulent in Se-deficient or vitamin E-deficient animals (Beck et al. 1994b). In addition, this change in virulence has been found to be due to specific mutations in the virus itself, such that once the mutations occurred, even mice with normal nutrition become vulnerable to the virus (Beck et al. 1995).

Our work has important implications for the effect of malnutrition on viral infection. If our work is applicable beyond coxsackieviruses, then it suggests that the nutritional status of the host is an important consideration for understanding the development of viruses with new pathogenic properties.

\section{Keshan disease}

Our interest in the relationship between infectious disease and host nutritional status began with studying Keshan 
disease (KD). KD is an endemic cardiomyopathy first described in China in the 1930s (Ge et al. 1983; Li et al. 1985). The pathology of KD is characterized by necrotic lesions scattered throughout the myocardium (Gu, 1983). $\mathrm{KD}$ is found only in regions of China with Se-poor soils, and occurs only in individuals with low Se status. Children and women of child-bearing age living in endemic areas are at highest risk. Supplementation of populations living in low-Se areas with Se can prevent the disease (Keshan Disease Research Group of the Chinese Academy of Medical Sciences, 1979). However, Se deficiency alone does not appear to be sufficient to cause KD; the disease has a seasonal and annual incidence and not all Se-deficient individuals develop KD. Thus, an infectious cofactor has been suggested (Su et al. 1979; Bai et al. 1980; Li et al. 1995). Coxsackieviruses were already known to cause myocarditis, and scientists in China have been able to isolate enteroviruses from the blood and tissue of KD victims.

In order to study the relationship between coxsackievirus infection and myocarditis, we utilized a well-characterized mouse model of coxsackievirus-induced myocarditis. The disease in mice closely mimics the human course of myocarditis and is widely accepted as an appropriate animal model. To determine the effect of a Se deficiency on the development of myocarditis, mice were fed on a diet deficient or adequate in Se for 4 weeks before infection with coxsackievirus. We used two different strains of coxsackievirus B3 for the infection. One strain, CVB3/20, is myocarditic in mice. The other strain, CVB3/0, is a benign strain of virus which does not cause myocarditis. Both strains will replicate in heart tissue, but only the CVB3/20 virus induces myocarditis.

As shown in Fig. 1, Se-deficient mice infected with CVB3/20 developed much more severe myocarditis when compared with infected Se-adequate mice. Thus, the pathogenicity of the virus was increased in Se-deficient animals. Se-adequate mice infected with the benign CVB3/0 did not

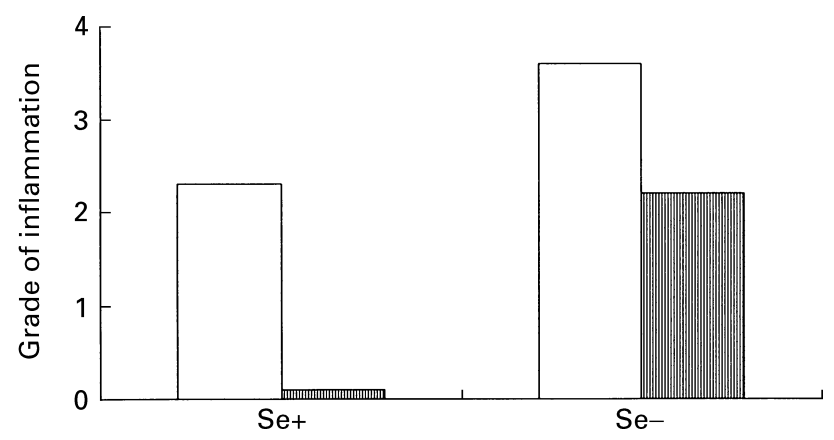

Fig. 1. Effect of selenium status on the development of myocarditis. Mice were fed on a diet either adequate $(\mathrm{Se}+)$ or deficient $(\mathrm{Se}-)$ in selenium for 4 weeks, then infected with either CVB3/20 (myocarditic strain; $\square$ ) or CVB3/0 (amyocarditic strain; mim). Heart pathology was scored at $10 \mathrm{~d}$ post infection for pathology as follows: 0, no lesions; 1 , foci of mononuclear cell inflammation associated with myocardial cell reactive changes without myocardial cell necrosis; 2, inflammatory foci clearly associated with myocardial cell reactive changes; 3 , inflammatory foci clearly associated with myocardial cell necrosis and dystrophic calcification; 4, extensive inflammatory infiltration, necrosis and dystrophic calcification. Values are means for ten animals. Standard deviations did not exceed 0.3 for any group. develop any myocarditis, as expected, whereas Se-deficient mice developed moderate myocarditis (Fig. 1). Thus, the virulence of the normally-benign virus was altered in the Se-deficient animals. Clearly, a deficiency in Se increased the pathogenicity of CVB3 virus; a normally-virulent strain caused increased myocarditis and a normally-benign virus induced myocarditis (Beck et al. 1994a,c).

The next question to be answered was whether the deficiency in Se affected the rate or titre of virus replication. When mice are inoculated intraperitoneally with the virus, shortly after infection the virus replicates in the liver. Following this initial round of viral replication in the liver, the virus spreads to the heart and another round of replication occurs in this organ and, in the case of the virulent strain of virus (CVB3/20), culminates in myocarditis. We found that Se-deficient mice had increased virus titres in both the heart and liver at 3, 5, 7 and $10 \mathrm{~d}$ post infection for both CVB3/0 and CVB3/20 viruses. Although viral titres were elevated in the Se-deficient mice, the Se-deficient mice were able to clear the virus by day 14 post infection. This rate of clearance is similar to that occurring in Se-adequate mice. Thus, although higher viral loads occurred in the infected Se-deficient mice, the ability of the Se-deficient mice to eventually clear the virus was not altered.

\section{Immune response}

A deficiency in Se has been reported by other workers to affect the immune system and to affect the ability to respond to infection (Harbige, 1996; Chen et al. 1997; Kukreja \& Khan, 1998). Thus, a decrease in immune responsiveness could be responsible for the changes in viral pathogenicity found in the Se-deficient mice. In order to determine the status of the immune response of the Se-deficient mice, we measured both T-cell and B-cell functions.

Production of neutralizing antibody responses are an important component of the immune response to viral infection and help to protect against re-infection with the virus. We found no differences in neutralizing antibody titres of infected mice whether they were fed on a diet sufficient or deficient in Se (Beck et al. 1994a,b). Thus, the deficiency in Se did not alter this component of the immune response.

To test T-cell function we cultured splenocytes from Se-deficient and Se-adequate mice $10-14 \mathrm{~d}$ post infection with a specific antigen as well as a mitogen, concanavalin A. We found that the proliferative response to both the viral antigen and the mitogen were greatly diminished in the Se-deficient animals when compared with the Se-adequate mice. This reduced response was found for either CVB3/0 or CVB3/20 infection. Thus, although the ability to produce neutralizing antibodies was not affected by the Se deficiency, the ability of T-cells to respond to antigen or mitogen in vitro was profoundly affected.

Since Se-deficient mice had deficient T-cell proliferative responses, we reasoned that the production of cytokines might also be compromised in the Se-deficient mice. Production of cytokines is an important feature of CVB3-induced myocarditis. We found that this component of the immune system was also altered in the Se-deficient mice; examination of heart-draining lymph nodes from 
Se-deficient mice demonstrated a decrease in mRNA levels for both interferon- $\gamma$ and interleukin 2 when compared with Se-adequate mice (MA Beck and OA Levander, unpublished results).

\section{Viral mutation}

There are two possibilities that could explain the change in virulence of the normally-benign virus in the Se-deficient mice. The first explanation is that because the immune response of the Se-deficient mice was impaired, the virus was able to replicate to a higher titre in the heart and thus cause heart damage. In a Se-adequate animal with a normal immune response no myocarditis occurred, because the viral titres did not reach a level at which myocarditis was induced. A second possibility is that the virus itself may have been altered due to replication in a Se-deficient host; i.e. the virus itself mutated to a virulent phenotype in the Se-deficient animal. In order to distinguish between these two possibilities, we designed a viral passage experiment (Beck et al. 1995). In this experiment virus isolated from CVB3/0 infected mice is passed into Se-adequate mice. If the change in virulence is due entirely to host factors, then the Se-adequate mice infected with the passed virus will not develop myocarditis. However, if the virus itself is altered as a consequence of replicating in a Se-deficient host, then the Se-adequate mice would not be protected from the passed virus, and will develop myocarditis.

For the passage experiment CVB3/0 virus was inoculated into Se-deficient and Se-adequate mice. At $7 \mathrm{~d}$ post infection virus was isolated from the hearts of both groups of mice. The virus was then redesignated to reflect the host it had been isolated from; virus obtained from Se-deficient mice was termed CVB3/0Se- and virus obtained from Se-adequate mice was termed CVB3/0Se+. CVB3/0Se- and $\mathrm{CVB} 3 / 0 \mathrm{Se}+$ viruses were re-inoculated into Se-adequate mice. Se-adequate mice infected with $\mathrm{CVB} 3 / 0 \mathrm{Se}+$ virus did not develop any myocarditis, demonstrating that viral passage alone did not change the phenotype of the virus. However, Se-adequate mice infected with CVB3/0Se- virus developed myocarditis, demonstrating that a phenotype change had occurred in the virus that replicated in a Se-deficient host. Thus, these results indicate that the virus itself had changed due to replication in a Se-deficient host.

In order to confirm that the phenotype change of the virus was due to a change in the viral genotype, we sequenced both the CVB3/0Se- virus and the CVB3/0Se+ virus. We found six nucleotides were altered in the CVB3/0Se- virus: nucleotide nos. $234(\mathrm{C} \rightarrow \mathrm{T}), 788(\mathrm{G} \rightarrow \mathrm{A}), 2271(\mathrm{~A} \rightarrow \mathrm{T})$, $2438(\mathrm{G} \rightarrow \mathrm{C}), 3324(\mathrm{C} \rightarrow \mathrm{T})$ and $7334(\mathrm{C} \rightarrow \mathrm{T}$; Beck et al. 1995). These changes lie in both non-translated and translated regions of the virus. All six of these nucleotide changes are found in other virulent strains of CVB3 viruses. No changes were found in the CVB3/0Se+ virus. This virus remained identical to the parent CVB3/0 strain. Thus, we demonstrated for the first time that replication of a normally-benign virus in a Se-deficient host can alter the genotype of the pathogen, such that a change in virulence occurs. Once these mutations occur, even mice of normal Se nutriture can be affected. The mechanism for the viral genotype change is not known. However, one possibility is that the oxidative stress status of the host was involved in affecting the viral genome.

\section{Oxidative stress}

Se is an essential component of the peroxide-destroying enzyme, GSH peroxidase (EC 1.11.1.9; GPX); therefore, one function of $\mathrm{Se}$ is its role as an antioxidant. To determine if other antioxidants would also have an effect on the virus, we investigated the effect of vitamin $E$ in our system. Vitamin $E$ is a lipid-soluble vitamin that acts as a free radical scavenger. Thus, similar to Se, it works as an antioxidant, although by a very different mechanism. Furthermore, individuals living in areas where $\mathrm{KD}$ is endemic were also found to have marginal vitamin $\mathrm{E}$ status, in addition to being deficient in Se.

We found that mice fed on a diet deficient in vitamin $\mathrm{E}$ developed myocarditis when infected with CVB3/0, a response similar to that found in the Se-deficient mice (Beck et al. 1994b). The addition of fish oil (menhaden oil) to the vitamin E-deficient diet increased the pathology of the virus post infection (Fig. 2). Fish oil is a known vitamin $\mathrm{E}$ antagonist, and therefore acts as a pro-oxidant.

Viral titres were also elevated in the vitamin E-deficient mice, although again, as in the Se-deficient mice, the mice were able to clear the virus. The immune system of the vitamin E-deficient mice was also affected; both mitogen and antigen responses were decreased, although neutralizing antibody responses were similar for vitamin E-deficient and adequate mice.

We have also found that feeding excess Fe to mice allows the benign CVB3/0 virus to cause myocarditis in these animals (Beck \& Levander, 1998). Excess Fe in the liver is

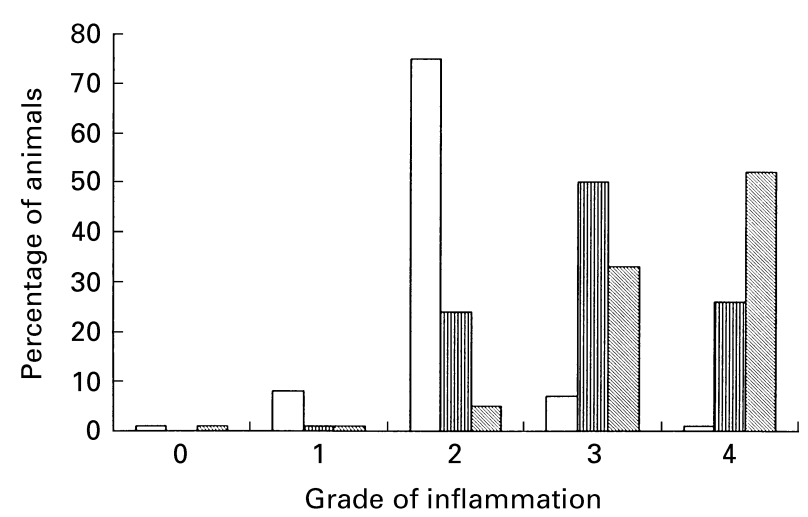

Fig. 2. Effect of vitamin $E$ status with or without the addition of fish oil on the development of myocarditis. Mice were fed on diets which were adequate $(\square)$ or deficient (IIII) in vitamin $E$ and without fish oil, and a vitamin E-deficient diet with menhaden oil (חw) for 4 weeks before infection with CVB3/0 (amyocarditic strain). At $10 \mathrm{~d}$ post infection, heart pathology was scored as follows: 0 , no lesions; 1 , foci of mononuclear cell inflammation associated with myocardial cell reactive changes without myocardial cell necrosis; 2, inflammatory foci clearly associated with myocardial cell reactive changes; 3 , inflammatory foci clearly associated with myocardial cell necrosis and dystrophic calcification; 4, extensive inflammatory infiltration, necrosis and dystrophic calcification. Values shown represent twenty mice per dietary group. 
associated with increased hepatic liver peroxidation. CVB3 replicates in the liver before replication in the heart. Thus, the increased oxidative stress status of the liver may have affected the virus before its replication in cardiac tissue. Currently, we are sequencing the virus obtained from mice with Fe overload to determine whether nucleotide changes occurred in the virus.

All our observations taken together suggest a common mechanism of oxidative stress. Our working hypothesis is that increased oxidative stress of the host leads to increased mutations in the viral genome, which in turn leads to a change in viral virulence. This hypothesis suggests that Se deficiency of the host induces the viral mutations due to a decrease in the activity of the Se-containing antioxidant enzyme GPX. In order to test this hypothesis, we used a knockout mouse model.

\section{GSH peroxidase 1-knockout mice}

GPX-1-knockout (KO) mice and wild-type mice were inoculated with CVB3/0. We found that approximately half the GPX-1-KO mice developed myocarditis, whereas none of the wild-type mice developed any myocarditis (Beck et al. 1998). Cardiac viral titres were identical between wild-type and GPX-1-KO mice, although Se-deficient mice had higher viral titres when compared with Se-adequate mice. Interestingly, the immune response of the GPX-1-KO mice was not identical to the immune response of the Se-deficient mice. GPX-1-KO mice had normal proliferative responses to both mitogen and antigen, but greatly reduced antibody responses. This finding is in contrast to that for the Se-deficient mice, which had diminished proliferative responses to mitogen and antigen, but a normal antibody response.

We sequenced viruses obtained from both wild-type and GPX-1-KO mice. We found seven nucleotide changes in the virus obtained from GPX-1-KO mice, and no changes in the virus obtained from wild-type mice. Six of the seven nucleotide changes were identical to the six changes found in the Se-deficient mice. Of particular note, no nucleotide changes were found in the virus obtained from GPX-1-KO mice which did not develop myocarditis. Myocarditis did not occur without the viral genomic changes, suggesting that some, if not all, the nucleotide changes are required for pathogenesis.

These results indicate that GPX-1 activity is required to protect the mice from CVB3/0-induced myocarditis. Taken together, all our findings support the idea that increased oxidative stress of the host can lead to changes in the viral genotype, changing an avirulent virus to a virulent one.

What is the mechanism for the effect of the oxidative stress on the viral genome? One possibility is direct damage to viral RNA by oxygen radicals. A decrease in antioxidant protection of the host due to nutritional deficiencies would lead to an increase in oxygen radicals. Although oxidative damage to DNA has been well documented, a similar effect on RNA has not been well studied. However, it seems possible that increased oxidative stress would lead to damage of the viral RNA, leading to mutations that would alter the pathogenic potential of the virus.
A second possibility is the selection of naturallyoccurring mutations. An RNA virus consists of a closelyrelated collection of mutants, termed quasispecies (Domingo \& Holland, 1994; Domingo et al. 1995). The sequence of an RNA virus is a consensus sequence based on a population. The nutritional deficiency of the host may shift the balance of the quasispecies such that a new virus sequence becomes the consensus sequence, which may have altered properties. The selection of a new consensus sequence may occur due to the decreased immune response of the deficient host, which allows for an increase in viral titre to occur. The increase in viral replication may allow one particular sequence to out-compete the co-existing sequences.

These two possibilities are not mutually exclusive. Both selection and direct mutation of the virus in the deficient host may occur. Further research is required to distinguish between these possibilities.

\section{Optic and peripheral neuropathy in Cuba}

Are there other examples beyond KD that suggest that the oxidative stress status of the host affects a viral genome? One possibility is an epidemic of optic and peripheral neuropathy that occurred in Cuba in the early 1990s (Cuba Neuropathy Field Investigation Team, 1995). The illness was associated with an unbalanced diet that was low in animal protein, fat, B-group and other vitamins, and an increase in the consumption of sugar. Impairment of antioxidant pathways also occurred, as patients had low levels of riboflavin, vitamin $\mathrm{E}, \mathrm{Se}, \alpha$ - and $\beta$-carotenes and the carotenoid lycopene. Smoking was also a risk factor, which is thought to cause injury through oxidative damage.

Similar to KD, the involvement of a viral cofactor in addition to the nutritional deficiencies has been suggested. Enterovirus-like viruses were isolated from the cerebrospinal fluid of $85 \%$ of patients with neuropathy, compared with an isolation rate of $2 \%$ from patients without neuropathy (Mas et al. 1997). This enterovirus-like virus was found to have atypical growth characteristics in vitro, and was found to be antigenically related to both coxsackievirus A9 and B4. Western blot experiments demonstrated a lack of the typical capsid proteins that characterize an enterovirus.

Sequencing of this atypical virus in our laboratory revealed close homology with coxsackievirus A9. However, specific mutations in the virus genome in the area of the viral protease were found. These mutations are not found in any other enterovirus for which sequence data is available. The protease is responsible for cleavage of the viral capsid proteins, and a mutation in this area, which may affect the functioning of this enzyme, would be expected to affect the viral capsid. This finding might explain the lack of typical coxsackievirus capsid proteins found by Western blot.

We hypothesize that the epidemic of neuropathy in Cuba may have been due to the emergence of a new viral strain of coxsackievirus A9 which arose as a consequence of replication in a nutritionally-compromised host. We are currently actively sequencing other viral isolates obtained from patients both with and without neuropathy. 


\section{Conclusions}

Our work points out the importance of a balance between pro-oxidant and antioxidant nutrition. The fact that increased oxidative stress of a host can lead to changes in a viral pathogen suggests that host nutritional status may be an important mechanism for the development of emerging viral pathogens with new pathogenic properties. Thus, rather than looking at nutritional stress affecting only the host, we propose a new model system which takes into account the possibility of the nutritional status of the host directly affecting the virus. This system can be illustrated as follows:

$$
\text { host malnutrition }
$$

increased oxidative stress<smiles></smiles>

decreased immunity $\Leftrightarrow$ altered viral genome

increased susceptibility to viral pathogenesis

This scheme takes into account the interplay between the nutritional status of the host, the immune response of the host, the virus itself, and the pathological outcome.

Further work is necessary in order to understand how applicable our findings are to other viruses. We believe that RNA viruses, like enteroviruses, may be more susceptible to oxidative damage than DNA viruses because of their lack of proof-reading enzymes (Steinhauer et al. 1992). Thus, genomic mistakes that occur in RNA viruses cannot be repaired during replication. In general, therefore, RNA viruses have a high mutation rate. Oxidative stress of the host could increase this mutation rate. Work is currently underway to determine whether the mutation rates of influenza viruses, rotaviruses and polioviruses are also influenced by the oxidative stress status of the host.

\section{References}

Bai J, Wu S, Ge K, Deng X \& Su C (1980) The combined effect of selenium deficiency and viral infection on the myocardium of mice. Acta Academy Medical Sinica 2, 31-33.

Beck MA, Esworthy RS, Ho Y-S \& Chu F-F (1998) Glutathione peroxidase protects mice from viral-induced myocarditis. FASEB Journal 12, 1143-1149.

Beck MA, Kolbeck PC, Rohr LH, Shi Q, Morris VC, Mas P, Pelegrino JL, Guzman MG, Comellas MM, Resìk S, Alva Rodrìguez $\mathrm{R}$, Mune $\mathrm{M}$, Capo $\mathrm{V}$, Balmaseda $\mathrm{A}$, Rodrìguez $\mathrm{L}$, Rodrìguez Handy J, Kouri G \& Llop A (1994a) Benign human enterovirus becomes virulent in selenium-deficient mice. Journal of Medical Virology 43, 166-170.

Beck MA, Kolbeck PC, Rohr LH, Shi Q, Morris VC, Mas P, Pelegrino JL, Guzman MG, Comellas MM, Resìk S, Alva Rodrìguez $\mathrm{R}$, Mune $\mathrm{M}$, Capo $\mathrm{V}$, Balmaseda $\mathrm{A}$, Rodrìguez L, Rodrìguez Handy J, Kouri G \& Llop A (1994b) Vitamin E deficiency intensifies the myocardial injury of coxsackievirus B3 infection in mice. Journal of Nutrition 124, 345-358.

Beck MA, Kolbeck PC, Shi Q, Rohr LH, Morris VC, Mas P, Pelegrino JL, Guzman MG, Comellas MM, Resìk S, Alva
Rodrìguez R, Mune M, Capo V, Balmaseda A, Rodrìguez L, Rodrìguez Handy J, Kouri G \& Llop A (1994c) Increased virulence of a human enterovirus (coxsackievirus B3) in selenium-deficient mice. Journal of Infectious Diseases 170, 351-357.

Beck MA \& Levander OA (1998) Dietary oxidative stress and the potentiation of viral infection. Annual Review of Nutrition 18, 93-116.

Beck MA, Shi Q, Morris VC \& Levander OA (1995) Rapid genomic evolution of a non-virulent coxsackievirus B3 in selenium-deficient mice results in selection of identical virulent isolates. Nature Medicine 1, 433-436.

Chen C, Zhou J, Zu H, Jiang Y \& Zhu G (1997) Effect of selenium supplementation on mice infected with LP-BM5 MuLY, a murine AIDS model. Biological Trace Element Research 59, 187-193.

Cuba Neuropathy Field Investigation Team (1995) Epidemic optic neuropathy in Cuba - Clinical characterization and risk factors. New England Journal of Medicine 333, 1176-1182.

Domingo E \& Holland JJ (1994) Mutation rates and rapid evolution of RNA viruses. In The Evolutionary Biology of Viruses, pp. 161-184 [SS Morse, editor]. New York: Raven Press.

Domingo E, Holland JJ, Biebricher C \& Eigen M (1995) Quasispecies: The concept and the word. In Molecular Evolution of the Viruses, pp. 171-180 [A Gibbs, C Calisher and F Garcia-Arenal, editors]. Cambridge: Cambridge University Press.

Ge KY, Xue A \& Bai J (1983) Keshan disease - an endemic cardiomyopathy in China. Virchows Archives 401, 1-14.

Gu BQ (1983) Pathology of Keshan disease. A comprehensive review. Chinese Medical Journal 96, 251-261.

Harbige LS (1996) Nutrition and immunity with emphasis on infection and autoimmune disease. Nutrition and Health 10, 285-312.

Keshan Disease Research Group of the Chinese Academy of Medical Sciences (1979) Observations on effect of sodium selenite in prevention of Keshan disease. Chinese Medical Journal 92, 471-476.

Kukreja R \& Khan A (1998) Effect of Se deficiency and its supplementation on DTH response, antibody forming cells and antibody titre. Indian Journal of Experimental Biology 36, 203-205.

Li Y, Wang F, Kang D \& Li C (1985) Keshan disease: an endemic cardiomyopathy in China. Human Pathology 16, 602-609.

Li Y, Yang Y \& Chen H (1995) Detection of the enteroviral RNA in paraffin-embedded myocardial tissue from patients with Keshan disease by nested PCR. Chung Hua I Hsueh Tsa 75, 344-345.

Mas P, Pelegrino JL, Guzman MG, Comellas MM, Resik S (1997) Viral isolation from cases of epidemic neuropathy in Cuba. Archives of Pathology and Laboratory Medicine 121, 825-833.

Scrimshaw N (1975) Nutrition and infection. Progress in Food Nutrition Science 1, 393-420.

Scrimshaw NS, Taylor CE, \& Gordon JE (1968) Interactions of Nutrition and Infection. WHO Monograph Series no. 57, Geneva: WHO.

Steinhauer D, Domingo E \& Holland JJ (1992) Lack of evidence for proofreading mechanisms associated with an RNA virus polymerase. Gene 122, 281-288.

Su C, Gong C, Li J, Chen L, Zhou D \& Jin Q (1979) Preliminary results of viral etiology of Keshan disease. Chinese Medical Journal 59, 466-472. 
